\title{
Commentary in Response to Adiponectin, Gut Hormones, and Insulin Resistance
}

\author{
Katrina L. Butner - Sharon M. Nickols-Richardson • \\ Susan F. Clark • Warren K. Ramp • William G. Herbert
}

Published online: 5 September 2010

(C) Springer Science+Business Media, LLC 2010

Our review included data from the recent research article published by Trakhtenbroit et al. [1], which was strengthened by the inclusion of patients followed for 2 years after undergoing either Roux-en-Y gastric bypass or adjustable gastric banding. Both our review [2] and the research by Trakhtenbroit et al. [1] came to similar conclusions and emphasized the need for long-term research following bariatric surgery. In addition, the authors suggested additional research regarding GLP1 as a pharmacological agent which could aid in the treatment of both type 2 diabetes and obesity. In the Letter to the Editor, the authors explained the exclusion criteria for their study [1] and noted that patients with diabetes were included. We appreciate the clarification and believe it adds value to the conclusions drawn in their research study.

We thank the authors for their letter and clarification of the exclusion criteria.

The authors declare that they have no conflict of interest.

\section{References}

1. Trakhtenbroit MA, Leichman JG, Algahim MF, et al. Body weight, insulin resistance, and serum adipokine levels 2 years after 2 types of bariatric surgery. Am J Med. 2009;122:435-42.

2. Butner KL, Nickols-Richardson SM, Clark SF, et al. A review of weight loss following roux-en-y gastric bypass vs restrictive bariatric surgery: impact on adiponectin and insulin. Obes Surg. 2010;20(5):559-68.

K. L. Butner $(\bowtie) \cdot$ W. G. Herbert

Laboratory for Health and Exercise Science and Bone,

Osteoporosis, Nutrition, and Exercise Laboratory, Department of

Human Nutrition, Foods and Exercise,

Virginia Polytechnic Institute and State University,

Blacksburg, VA, USA

e-mail: kbutner@vt.edu

S. M. Nickols-Richardson

Bone Metabolism, Osteoporosis, and Nutrition Evaluation

Laboratory, Department of Nutritional Sciences,

The Pennsylvania State University,

University Park, PA, USA

\section{S. F. Clark}

Department of Human Nutrition, Foods and Exercise,

Virginia Polytechnic Institute and State University,

Blacksburg, VA, USA

W. K. Ramp

Health Research Group, LLC,

Rockbridge Baths, VA, USA 